Neth. J. Pl. Path. 98 (1992) 203-212

\title{
Biological control of the leafminer Liriomyza bryoniae by seasonal inoculative releases of Diglyphus isaea: simulation of a parasitoid-host system
}

\author{
W.J. BOOT ${ }^{1}$, O.P.J.M. MINKENBERG ${ }^{2}$, R. RABBINGE ${ }^{1}$ and G.H. DE MOED ${ }^{1}$ \\ ${ }^{1}$ Department of Theoretical Production Ecology, Wageningen Agricultural University, P.O. \\ Box 430, 6700 AK Wageningen, the Netherlands \\ 2 Department of Entomology, Wageningen Agricultural University, P.O. Box 8031, 6700 \\ EH Wageningen, the Netherlands
}

Accepted 5 February 1992

\begin{abstract}
A deterministic model was developed to simulate population growth of the agromyzid fly Liriomyza bryoniae and the parasitoid Diglyphus isaea. The model has two driving variables, ambient temperature and leaf nitrogen content of the tomato plant. Results of a glasshouse experiment were used to validate the model. The timing of successive generations of leafminers was simulated accurately over four generations. Population growth of leafminers was correctly simulated during the first two generations, but overestimated in the third generation. Mortality of leafminers due to parasitism was overestimated in the first generation after introduction of parasitoids: $73 \%$ instead of the observed $30 \%$. A nearly $100 \%$ mortality of leafminers was correctly simulated in the second generation after introduction of parasitoids. Sensitivity analysis was performed for three types of variables: (1) driving variables, temperature and leaf nitrogen content; (2) parasitoid traits, searching efficiency and allocation of attacks to host feeding and oviposition, and (3) introduction strategies for biological control, timing, number of releases and number of parasitoids per release. Population growth was sensitive to temperature, leaf nitrogen content, searching efficiency of parasitoids and numbers of parasitoids released.
\end{abstract}

Additional keywords: Liriomyza bryoniae, Diglyphus isaea, simulation model, biological control, parasitoid-host interactions.

\section{Introduction}

The agromyzid fly Liriomyza bryoniae (Kaltenbach) is an important pest in European greenhouses (Minkenberg \& Van Lenteren, 1986). The larvae, leafminers, feed in the mesophyll tissue of various vegetables and may thereby reduce quality and yield (Spencer, 1973). Several parasitoids have been evaluated for seasonal inoculative releases on greenhouse tomatoes and a biological control method has been developed (reviewed in Minkenberg \& Van Lenteren, 1986).

Most biological control agents are found by trial and error (Van Lenteren, 1986). Explanation of the effectiveness of an agent often happens in hindsight. To help in the development of insight on the selection criteria for biological control agents, a 
population dynamical model was developed, thus bridging the gap between results from field introductions and data from detailed laboratory studies on the life history of L. bryoniae and their ectoparasitoid Diglyphus isaea on tomato. Heinz et al. (1990) recently presented a simulation model for another leafminer pest, Liriomyza trifolii.

\section{Methods}

The model was constructed using general simulation principles in crop protection (Rabbinge, 1976; Rabbinge et al., 1989).

Driving variables. The model has two driving variables, ambient temperature and leaf nitrogen content of the plant. Temperature affects almost all biological processes in the model, such as immature development, immature mortality, female longevity and reproduction (Fig. 1A, B, C, E, F; Minkenberg \& Helderman, 1990; Minkenberg, 1989). Leaf nitrogen content affects only a few life history parameters of the host: at higher nitrogen levels the duration of immature development and immature mortality are decreased, whereas female longevity and fecundity are increased (Fig. 1D). Effects of leaf nitrogen content on life history parameters were inferred from a study on $L$. trifolii, a species closely resembling $L$. bryoniae (Minkenberg \& Ottenheim, 1990). No other interaction between leafminers and their host plant, which is regarded as a constant source of food, is modelled. The two driving variables are assumed to act instantaneously and independently.

Development, ageing and reproduction of L. bryoniae. Development of each leafminer instar is modelled using a boxcar train method, in which relative dispersion of development time per instar is temperature dependent (De Wit \& Goudriaan, 1978; Goudriaan \& Van Roermund, 1989). Immature mortality is considered proportional to the actual number of individuals and is represented by a relative mortality rate per instar. Mortality and reproduction of females strongly depend on age. Ageing of females is modelled using a boxcar train method without dispersion (De Wit \& Goudriaan, 1978; Goudriaan \& Van Roermund, 1989): females are represented by ten age classes, each representing one tenth of the maximum longevity. Maximum longevity is defined as the average longevity plus three times its standard deviation, which is a good estimate when average longevity is normally distributed. In the model, the relative mortality rate and relative reproduction rate vary between classes, but are constant within each class. Relative reproduction rates per class are derived from the relation between age and reproduction (Fig. $1 \mathrm{~F}$ ). Relative mortality rates per class are calculated by treating survival curves as dose-response relations, where dose is age (days) and response is death (Clark, 1987). Average longevity of females could be described by a normal distribution and therefore the relative mortality rate for each class is calculated using the normal probability density function; see Appendix.

Development, ageing and reproduction of $D$. isaea. To model development, ageing and reproduction of parasitoids the same methods are used as described for the host, except for interactions between leafminers and parasitoids. The rate of successful attacks of parasitoids, i.e. resulting in oviposition or host feeding, is calculated with a 'type 2' functional response (Holling, 1959). 


\section{$\mathrm{RATT}=\mathrm{A} * \mathrm{DENS} * \mathrm{RMAX} /(\mathrm{A} * \mathrm{DENS}+\mathrm{RMAX})$}

where, RATT $=$ Per capita rate of successful attacks (larvae/day), A = Searching efficiency (plants/day), DENS = Density of larvae (larvae/plant), RMAX = Per capita maximum rate of successful attacks (larvae/day; Fig. 1E).

An accurate estimate of the searching efficiency is not available. The order of magnitude was estimated to be one plant per day, based on estimates of the rate of successful attacks (RATT), density of larvae (DENS) and the maximum rate of successful attacks (RMAX). Host feeding results in mortality of the host, whereas oviposition results in mortality of the host and in reproduction of the parasitoid. Therefore, successful attacks are divided into oviposition and host feeding using a fixed oviposition proportion of 0.65 , as laboratory experiments showed that $50 \%$ to $75 \%$ of the successful attacks concerned oviposition (Minkenberg, 1989). First instars of the leafminers are used for host feeding only and not for oviposition. Consequently, the average probability of successful attacks in the host population allocated to oviposition falls below 0.65 when first instars are abundant.

Validation of the model. Results of a glasshouse experiment conducted by Westerman \& Minkenberg (1986) were used to validate the model. A small glasshouse was planted with 390 tomato plants and infested with 30 third instar larvae of $L$. bryoniae. About 200 female parasitoids, $D$. isaea, were introduced as soon as the first leafminers of the third generation appeared. Temperature was recorded every hour, but leaf nitrogen content of the plants was not determined. A value of $6 \%$ (dry weight) was used in the model, which yields the temperature dependant life history variables found by Minkenberg \& Helderman (1990; Fig. 1D). Westerman \& Minkenberg (1986) counted falling pupae of $L$. bryoniae under one tenth of the plants using nets. Their data indicate at which moment consecutive host generations are present. Net reproduction of $L$. bryoniae (new mines/mine per generation), derived from mine counts and corrected for parasitism, was used to validate population growth of leafminers.

\section{Results}

Validation of the model in absence of parasitoids. Peak numbers of observed pupations per day were found at day 44, day 71, 107, 135 in the first, second, third and fourth generation of $L$. bryoniae, respectively. Peak numbers of simulated pupations were on day $41,73,104$ and 136, respectively (Fig. 2). Thus the timing of generations was correctly simulated. During the first two generations, observed and simulated net reproduction were similar, with 59 and 54 new mines/mine in the first, and 33 and 33 new mines/mine in the second generation, respectively. The observed net reproduction in the third generation was 13 , which was lower than the simulated 33 new mines/mine.

Validation of the model in presence of parasitoids. Direct validation of the parasitoid part of the model was not possible, as parasitoid counts had not been made. For indirect validation the number of host pupated per day could be used (Fig. 2), but Westerman \& Minkenberg (1986) found pupal sampling to be unreliable as a measure Neth. J. Pl. Path. 98 (1992) 

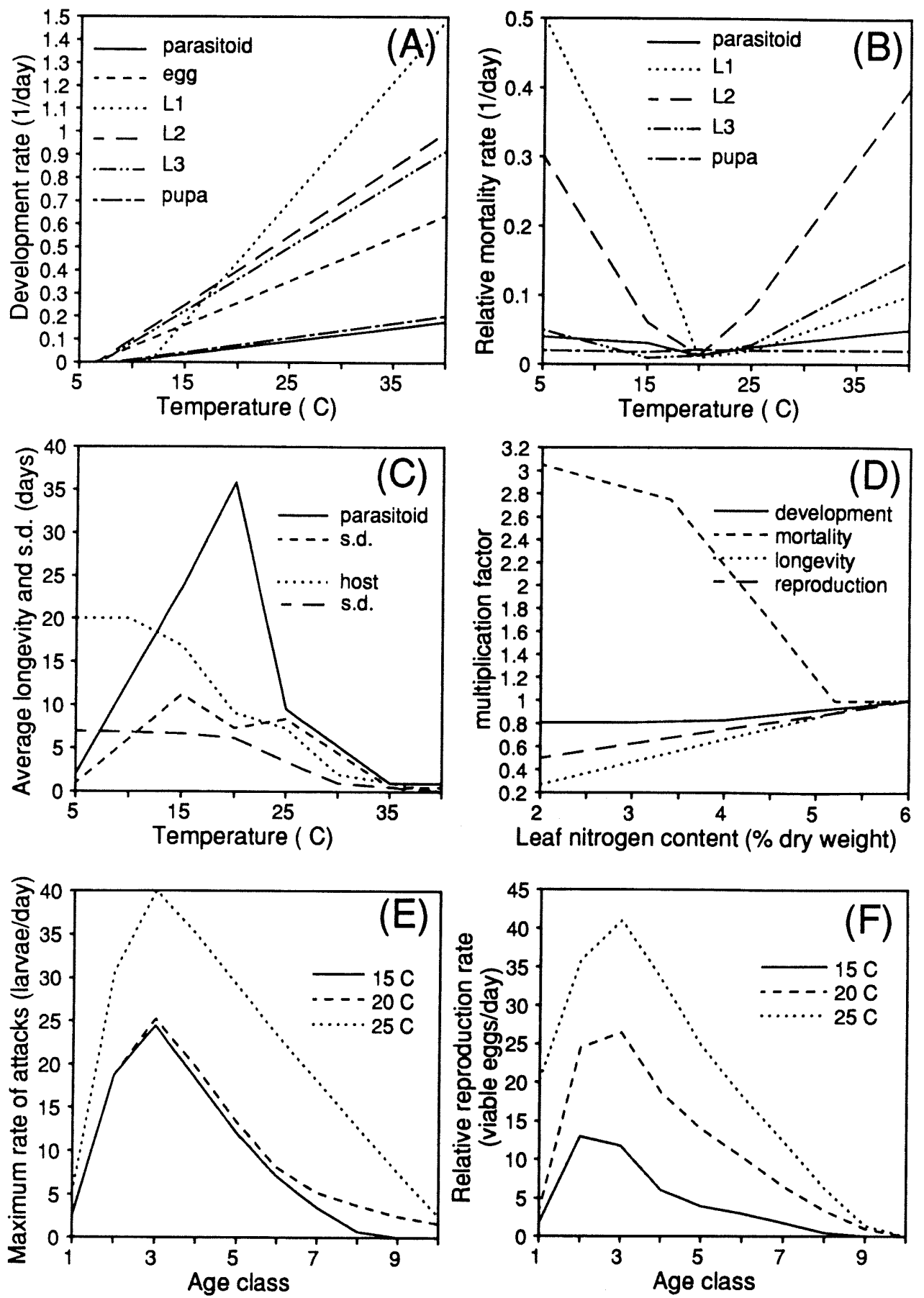

Fig. 1. Input relations of the model.

(A). Development rate of immature $D$. isaea (parasitoid) and $L$. bryoniae eggs (egg), first 206

Neth. J. Pl. Path. 98 (1992) 
instar larvae (L1), second instar larvae (L2), third instar larvae (L3) and pupae (pupa), as a function of temperature.

(B). Relative mortality rate of immature $D$. isaea (parasitoid) and $L$. bryoniae eggs (egg), first instar larvae (L1), second instar larvae (L2), third instar larvae (L3) and pupae (pupa), as a function of temperature.

(C). Average longevity and its standard deviation of female $D$. isaea (parasitoid longevity; s.d. of parasitoid longevity) and female $L$. bryoniae (host longevity; s.d. of host longevity) as a function of temperature.

(D). Factor by which the development rate of immature stages (development), the relative mortality rate of larval stages (mortality), the average longevity and its standard deviation of females (longevity), and the relative reproduction rates of females (reproduction) of $L$. bryoniae are multiplied, as a function of leaf nitrogen content.

(E). Maximum rate of successful attacks per female $D$. isaea at $15^{\circ} \mathrm{C}, 20^{\circ} \mathrm{C}$ and $25^{\circ} \mathrm{C}$ as a function of age.

(F). Relative reproduction rate of $L$. bryoniae at $15^{\circ} \mathrm{C}, 20^{\circ} \mathrm{C}$ and $25^{\circ} \mathrm{C}$ as a function of age.

of population growth. Therefore observed mortality of leafminers due to parasitism was used. Both observed and simulated mortality of leafminers were almost $100 \%$ in the second generation after introduction ( $99 \%$ and $100 \%$, repectively) but observed mortality was lower than simulated in the first generation after introduction $(30 \%$ and $73 \%$, respectively).

Sensitivity analysis. Sensitivity analysis shows which parameters are important in the population dynamics of a system, even though validation of the model is still incomplete. The effect of driving variables, parasitoid traits and introduction strategies on model output were evaluated.

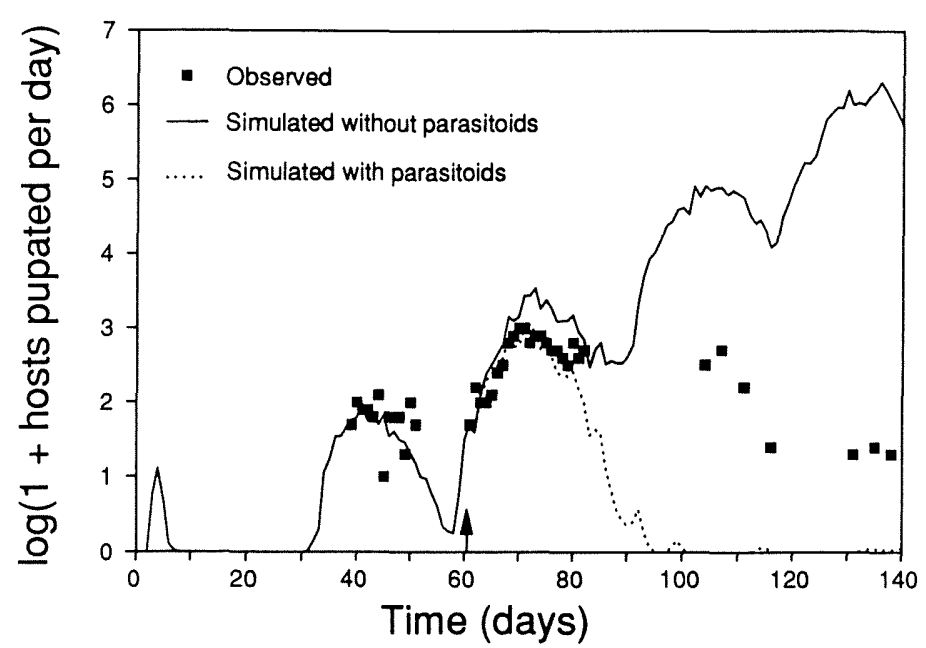

Fig. 2. Number of L. bryoniae pupated per day observed (Westerman \& Minkenberg, 1986) and simulated (with and without introduction of parasitoids). Time 0 corresponds with 8 February 1986 in their study. Simulation was started at time is 0 with 30 third instar leafminers with temperatures as measured in the glasshouse and with leaf nitrogen content set at $6 \%$ of dry weight. 200 parasitoids were introduced at time is 61 (arrow).

Neth. J. Pl. Path. 98 (1992) 
Table 1. Effect of temperature $\left({ }^{\circ} \mathrm{C}\right)$ on simulated cumulative number of mines after 150 days, net reproduction (no. of new mines/ mine per generation) and generation time (days) of a $L$. bryoniae population. The model was initialized with 100 eggs, temperature was given as a daily fluctuating sinusoidal function with an amplitude of $5{ }^{\circ} \mathrm{C}$ and leaf nitrogen content was set at $6 \%$ of dry weight.

\begin{tabular}{llll}
\hline $\begin{array}{l}\text { Average } \\
\text { temperature } \\
\left({ }^{\circ} \mathrm{C}\right)\end{array}$ & $\begin{array}{l}\text { Cum. no. } \\
\text { of mines }\end{array}$ & $\begin{array}{l}\text { Net } \\
\text { reproduction }\end{array}$ & $\begin{array}{l}\text { Generation } \\
\text { time }\end{array}$ \\
18 & $1.3 \times 10^{8}$ & 36.9 & 37 \\
19 & $7.4 \times 10^{8}$ & 44.4 & 35 \\
20 & $1.1 \times 10^{10}$ & 50.8 & 31 \\
21 & $3.9 \times 10^{10}$ & 54.0 & 29 \\
22 & $2.4 \times 10^{11}$ & 54.1 & 27 \\
\hline
\end{tabular}

Table 2. Effect of leaf nitrogen ( $\%$ dry weight) on simulated cumulative number of mines after 150 days, net reproduction (no. of new mines/ mine per generation) and generation time (days) of a $L$. bryoniae population. The model was initialized with 100 eggs and temperature was given as a daily fluctuating sinusoidal function with an average temperature of $20^{\circ} \mathrm{C}$ and an amplitude of $5^{\circ} \mathrm{C}$.

\begin{tabular}{llll}
\hline $\begin{array}{l}\text { \% leaf } \\
\text { nitrogen }\end{array}$ & $\begin{array}{l}\text { Cum. no. } \\
\text { of mines }\end{array}$ & $\begin{array}{l}\text { Net } \\
\text { reproduction }\end{array}$ & $\begin{array}{l}\text { Generation } \\
\text { time }\end{array}$ \\
3 & $4.3 \times 10^{5}$ & 6.7 & 34 \\
4 & $2.2 \times 10^{7}$ & 15.8 & 33 \\
5 & $1.5 \times 10^{9}$ & 36.0 & 32 \\
6 & $1.1 \times 10^{10}$ & 50.8 & 31 \\
\hline
\end{tabular}

Changes in driving variables. Increase in temperature strongly affects population growth of leafminers, due to a larger net reproduction and a shorter generation time (Table 1). The cumulative number of mines after 150 days at $22^{\circ} \mathrm{C}$ is 1800 times the number at $18{ }^{\circ} \mathrm{C}$. Differences of $1-2 \%$ in leaf nitrogen content, which are often found, affect population growth of leafminers also strongly, primarily by effects on net reproduction (Table 2). Generation time is only slightly affected by leaf nitrogen content, as faster larval development goes with prolongation of female longevity at higher nitrogen levels.

Changes in parasitoid traits. Sensitivity analysis of the searching efficiency of parasitoids was done using the same temperatures as measured in the glasshouse experiment (Fig. 3A). 200 female parasitoids were introduced at day 61 and leafminer density had reached ca. five mines per plant at that time. When the assumed searching efficiency of 1.0 plant/day was lowered to 0.5 plant/day, part of the leafminers escaped parasitism in the second generation after parasitoid introduction. In the simulation escape from parasitism in the third generation after parasitoid introduction was not found. 


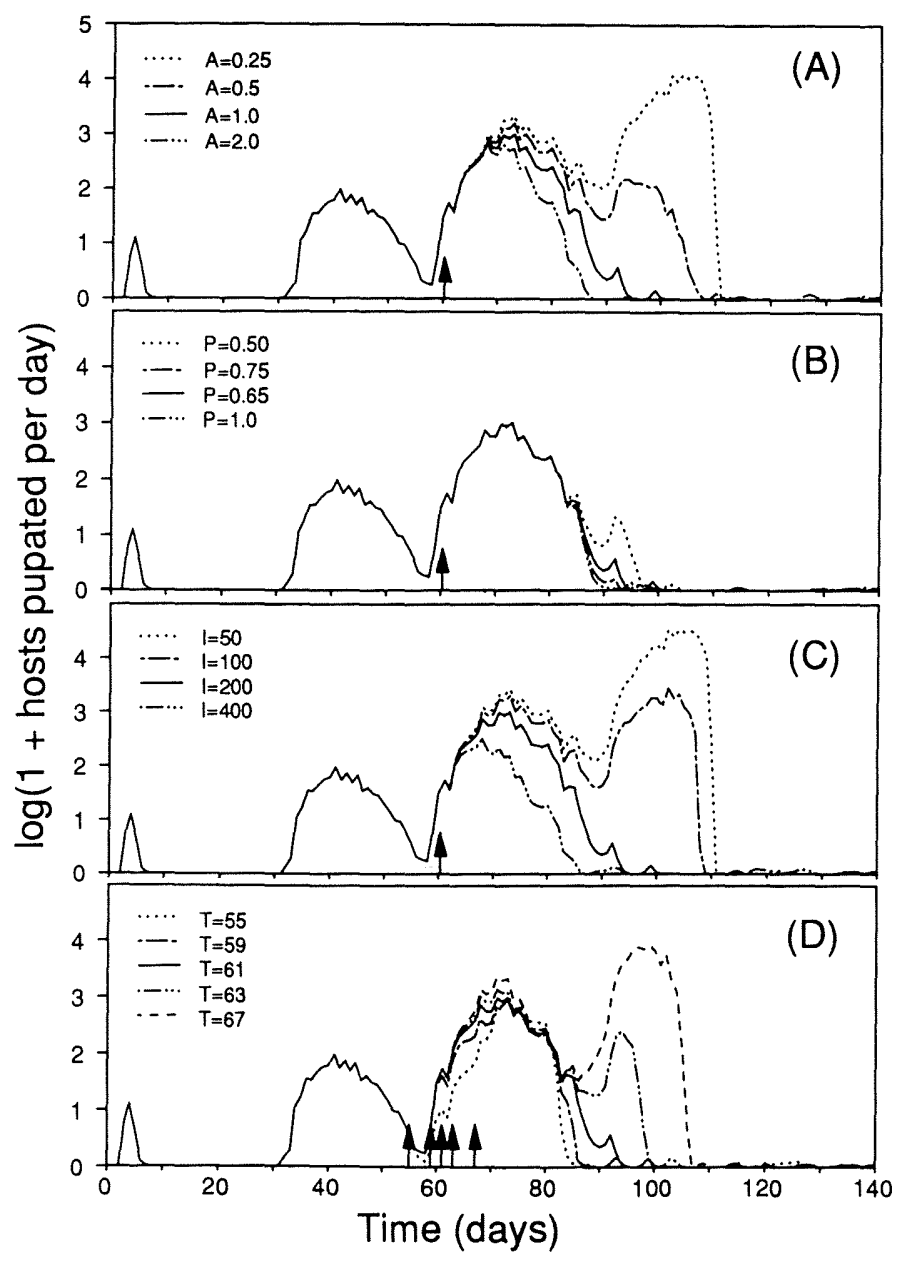

Fig. 3. Simulated number of $L$. bryoniae pupated per day. The model was initialised with 30 third instars, temperatures were as measured in the glasshouse experiment, total leaf nitrogen content was set at $6 \%$ of dry weight and 200 female parasitoids were introduced (arrows).

(A). With searching efficiency (A) of $D$. isaea of $0.25,0.5,1.0$ and 2.0 plants/day.

(B). With probability of successful attacks allocated to oviposition (P) of $0.50,0.65,0.75$ and 1 .

(C). With number of introduced female $D$. isaea (I) of 50,100, 200 and 400 parasitoids.

(D). With time of introduction of $D$. isaea (T) on day 55, day 59, day 61, day 63 and on day 67 .

Sensitivity to the percentage of attacks allocated to oviposition was low (Fig. 3B), probably because it only affects the number of parasitoids in the next generation and because total control was quickly achieved.

Changes in introduction strategies. Escape from parasitism is affected by the number of parasitoids introduced. According to the simulation model introduction with 200 female parasitoids into the experimental glasshouse was required, to prevent

Neth. J. Pl. Path. 98 (1992) 
Table 3. Simulated cumulative number of mines of a $L$. bryoniae population after total control* by parasitoids; 200 parasitoids were introduced simultaneously at day 61 or divided over 1 week intervals.

\section{Introduction scheme}

200 at day 61

100 at day 54 and 100 at day 61

100 at day 61 and 100 at day 68

67 at day 54, day 61 and day 68

67 at day 61 , day 68 and day 75
Cum. no. of mines
$0.60 \times 10^{6}$
$1.04 \times 10^{6}$
$1.91 \times 10^{6}$
$5.17 \times 10^{6}$
$8.35 \times 10^{6}$ * In the simulation, introduction of parasitoids leads to extinction of leafminers within 3 gene-
rations.

part of the leafminers from escaping parasitism in the second generation following the release (Fig. 3C). Introduction of 200 females was equivalent to ca. one female parasitoid per ten mines. As long as parasitoids were introduced before most leafminers had pupated, time of introduction was not very critical (Fig. 3D).

Introducing parasitoids only once provided a slightly better control than the schemes with weekly introductions (Table 3). Leafminer generations were still distinct at the time of introduction, both in the experiment and in simulations. Because suitable instars for parasitism were present for only a short period, repeated introductions did not enhance control.

\section{Discussion}

Timing of generations of leafminers and population growth in the first two generations were correctly simulated. Population growth was overestimated in the third generation. Host plant quality is probably not constant during the season, as assumed in the model, and may have affected population growth, for instance through changing levels of leaf nitrogen content (Minkenberg \& Ottenheim, 1990) and secondary plant compounds. Effects of leaf nitrogen content could not be validated, but sensitivity analysis showed that leaf nitrogen content can be important. The relation between population growth and leaf nitrogen content should be treated carefully, however, because it was inferred from a study on the effects of host plant quality on $L$. trifolii, a species closely resembling $L$. bryoniae (Minkenberg \& Ottenheim, 1990). Population growth was also sensitive to temperature, searching efficiency of parasitoids and number of parasitoids released.

Simulation of parasitism with only a few variables, as is done here, is attractive for its simplicity. However, these variables depend on many underlying processes, which are neglected in this model. For example, different responses of parasitoids to the three different instars of leafminers attacked (Hassell et al., 1976), emigration of parasitoids and a variable sex ratio of parasitoids (Charnov et al., 1981; Reeve, 1987; Heinz \& Parrella, 1990) may be important. Interaction between parasitoids is also an important phenomenon, as encounters between parasitoids may decrease their searching efficiency (Hassell, 1971; Hassell et al., 1976). Encounters with already parasitized hosts 
may have a similar effect. Moreover, interaction between leafminers and parasitoids may be affected by superparasitism and host feeding on parasitized hosts. Such interactions could easily occur in this parasitoid-host system, since parasitism was very high within a few generations. Thus, the actual interactions between leafminers and parasitoids and the mutual interactions between parasitoids are largely unknown. This does not preclude simulation, however, as long as an equation of the functional response can be reliably described from experimental results. Another possibility is to develop a detailed model at the level of the individual, which explicitly simulates the interactive processes between hosts and parasitoids. Such a model based on principles of behaviour, could be used to derive simplified relations for the parasitoid-host interactions, which could be used in a population model instead of the analytical function for functional response.

The present model shows, based on life history parameters found in laboratory studies, that seasonal inoculative releases of $D$. isaea might be successfully used against $L$. bryoniae.

\section{Appendix}

Mortality in a group of females having the same age $t$, can be calculated using the normal probability density function:

$$
\operatorname{Mort}(t)=\operatorname{IFEM} * 1 / \sigma *(2 * \pi)^{-0.5} * \mathrm{e}^{-0.5 *[(t-\mu) / \sigma]^{2}}
$$

where, $t=$ age (days), Mort $(t)=$ Mortality rate (females/day), $\mu=$ Average longevity (days), $\sigma=$ Standard deviation of the average longevity (days), IFEM = Initial number of females in the age group.

The relative mortality rate, $\operatorname{rmr}(t)$, can be calculated as the absolute mortality rate, $\operatorname{Mort}(t)$, divided by the number of females left, i.e. IFEM minus the integral of the probability density function for a normal distribution:

$$
\begin{aligned}
& \text { FEM }=\text { IFEM }- \text { IFEM } * \int_{0}^{t} 1 / \sigma *(2 * \pi)^{-0.5} * \mathrm{e}^{-0.5 *[(t-\mu) / \sigma]^{2}} \\
& \operatorname{rmr}(t)=\frac{\operatorname{Mort}(t)}{\operatorname{FEM}}
\end{aligned}
$$

FEM $=$ Number of females left, $\operatorname{rmr}(t)=$ Relative mortality rate $(1 /$ day $)$.

Thus, $\operatorname{rmr}(t)$ only depends on the average longevity $\mu$, its standard deviation $\sigma$ and age $t$, and is used to calculate the actual mortality of females present in the age classes. For each class of females the median age is used to calculate the relative mortality rate in it. 


\section{Acknowledgements}

We thank W. van der Werf and H.J.W. van Roermund for their valuable comments on earlier drafts of the manuscript.

\section{References}

Charnov, E.L., Los-Den Hartog, R.L., Jones W.T. \& Van den Assem, J., 1981. Sex-ratio evolution in a variable environment. Nature 289: 27-33.

Clark, A.G., 1987. The quantitative genetics of evolutionary constraints. In: V. Loeschke (Ed.), Genetic constraints on adaptive evolution. Springer-Verlag, Berlin: 188pp.

De Wit, C.T. \& Goudriaan, J., 1978. Simulation of ecological processes. Simulation Monographs, Pudoc, Wageningen: 175 pp.

Goudriaan, J. \& Van Roermund, H.J.W., 1989. Modelling of ageing, development, delays and dispersion. In: Rabbinge, R., Ward, S.A. \& Van Laar, H.H. (Eds), Simulation and systems management in crop protection. Pudoc, Wageningen: 47-79.

Hassell, M.P., 1971. Mutual interference between searching insect parasites. Journal of Animal Ecology 40: 473-486.

Hassell, M.P., Lawton, J.H. \& Beddington, J.R., 1976. The components of arthropod predation I. The prey death-rate. Journal of Animal Ecology 45: 135-164.

Heinz, K.M. \& Parrella, M.P., 1990. The influence of host size on sex ratios in the parasitoid Diglyphus begini (Hymenoptera: Eulophidae). Ecological Entomology 15: 391-399.

Heinz, K.M., Nunney, L. \& Parrella, M.P., 1990. Predictability of biological control of the leafminer Liriomyza trifolii, infesting greenhouse cut chrysanthemums. SROP/WPRS Bulletin XIII/5: 76-82.

Holling, C.S., 1959. The components of predation as revealed by a study of small-mammal predation of the European pine sawfly. Canadian Entomologist 91: 293-320.

Minkenberg, O.P.J.M. \& Van Lenteren, J.C., 1986. The leafminers Liriomyza bryoniae and Liriomyza trifolii (Diptera: Agromyzidae), their parasites and host plants: a review. Agricultural University Wageningen Papers 86-2: 1-50.

Minkenberg, O.P.J.M., 1989. Temperature effects on the life history of the eulophid wasp Diglyphus isaea, an ectoparasitoid of leafminers (Liriomyza spp.) on tomato. Annals of Aplied Biology 115: 381-397.

Minkenberg, O.P.J.M. \& Helderman, C.A.J., 1990. Effect of temperature on the life history of Liriomyza bryoniae (Diptera: Agromizidae) on tomato. Journal of Economic Entomology 83: $117-125$.

Minkenberg, O.P.J.M. \& Ottenheim, J.J.G.W., 1990. Effect of leaf nitrogen content of tomato plants on preference and performance of a leafmining fly. Oecologia 83: 291-298.

Rabbinge, R., 1976. Biological control of fruit-tree red spider mite. Simulation Monographs. Pudoc, Wageningen: $228 \mathrm{pp}$.

Rabbinge, R., Ward, S.A. \& Van Laar, H.H., 1989. Simulation and systems management in crop protection. Pudoc, Wageningen: 420 pp.

Reeve, J.D., 1987. Foraging behavior of Aphytis melinus: Effects of patch density and host size. Ecology 68: 530-538.

Spencer, K.A., 1973. Agromyzidae (Diptera) of economic importance. Series Entomologica, 9. W. Junk, The Hague: 418 pp.

Van Lenteren, J.C., 1986. Parasitoids in the greenhouse: successes with seasonal inoculative release systems. In: Waage, J.K. \& Greathead, D. (Eds), Insect parasitoids. Academic Press, Londen: $389 \mathrm{pp}$.

Westerman, P.R. \& Minkenberg, O.P.J.M., 1986. Evaluation of the effectiveness of the parasitic wasps Diglyphus isaea and Chrysocharis parksi in experimental greenhouses for the biological control of the leafminer Liriomyza bryoniae, on tomatoes. Meded. Fac. Landbouww. Rijksuniv. Gent 51: 999-1008. 